\title{
ABORDAGEM DIDÁTICA PARA O DESENVOLVIMENTO DE MOLÉCULAS BIOATIVAS: REGRA DOS CINCO DE LIPINSKI E PREPARAÇÃO DE HETEROCICLO 1,3,4-OXADIAZOL EM FORNO DE MICRO-ONDAS DOMÉSTICO
}

\author{
Victória Laysna dos Anjos Santos, Arlan de Assis Gonsalves e Cleônia Roberta Melo Araújo* \\ Colegiado de Ciências Farmacêuticas, Universidade Federal do Vale do São Francisco, Campus Petrolina Centro, 56304-917, \\ Petrolina - PE, Brasil
}

Recebido em 12/05/2017; aceito em 10/08/2017; publicado na web em 16/10/2017

\begin{abstract}
DIDACTIC APPROACH FOR THE DEVELOPMENT OF BIOATIVE MOLECULES: LIPINSKI'S FIVE-RULE AND PREPARATION OF 1,3,4-OXADIAZOL HETEROCYLIC IN DOMESTIC MICROWAVE OVEN. In Brazil, the development of new chemical entities (NEQ) is still limited to small advances in the academic and public research sectors, the authors understand that this is a sector of the pharmaceutical industry that needs encouragement. Accepting that education transforms society, educate students in the development of bioactive molecules, it's a way to stimulate the national pharmaceutical industry to invest in the sector. Thus, this work brings a didactic sequence, applicable in undergraduate courses, which exemplifies some stages of development of the NEQ through its rational planning. The synthesis of a 1,3,4-oxadiazole heterocycle was planned and its potentiality assessed through the Lipinski's Rule of Five (RO5), using the ChemSpider ${ }^{\circledR}$ database. Once in accordance with RO5, the heterocycle was prepared using a simple and accessible methodology, using microwaves for domestic use as heating source. The 2-phenyl-5-(pyridin-4-yl)-1,3,4oxadiazole was synthesized by oxidative cyclization of acylhydrazone and an overall yield of $90 \%$. So was possible to demonstrate in a practical way some steps for the development of a substance with potential biological activity and thus to attract greater interest of undergraduate students by this follow-up of the Medicinal Chemistry.
\end{abstract}

Keywords: Rule of five (RO5); Lipinski; new chemical entities (NEQ); domestic microwave oven; ChemSpider ${ }^{\circledR}$.

\section{INTRODUÇÃO}

Do ponto de vista econômico, as inovações podem se tornar o diferencial para a competitividade de empresas e países. Dentro deste cenário, a indústria farmacêutica é uma das mais lucrativas em razão da busca constante por produtos inovadores. Existem quatro estágios tecnológicos na indústria farmacêutica, sendo estes: Estágio I - Pesquisa e Desenvolvimento (P\&D); Estágio II - Produção de Farmoquímicos; Estágio III - Produção de Especialidades Farmacêuticas; e Estágio IV - Marketing e Comercialização das Especialidades Farmacêuticas. A descoberta de novas moléculas com potencial terapêutico (novas entidades químicas - NEQ) faz parte do Estágio Tecnológico I, uma atividade que compreende a pesquisa básica de screening, o desenho molecular e os estudos farmacológicos. ${ }^{1}$

No Brasil, tanto as indústrias nacionais quanto as subsidiárias de multinacionais atuam apenas nos Estágios Tecnológicos III e IV, ou seja, há formulação de medicamentos e comercialização, contudo, praticamente quase a totalidade das matérias-primas deve ser importada. Embora o país possua uma indústria de medicamentos bastante desenvolvida, sua capacidade tecnológica para gerar inovações ainda não é satisfatória, ficando as atividades de $\mathrm{P} \& \mathrm{D}$ basicamente restritas a setores acadêmicos e públicos de pesquisa. ${ }^{1}$

A Química Medicinal é uma área de estudo que visa o desenvolvimento de novos fármacos, e assim, fornece meios para a obtenção de NEQ. A síntese orgânica colabora com este processo promovendo a obtenção das moléculas propostas. A formação de recursos humanos capacitados para o desenvolvimento de NEQ é uma maneira de colaborar com o setor nacional de P\&D de fármacos, área considerada estratégica do ponto de vista social, econômico e político. Considerando que, de 70 a $80 \%$ do preço final de um medicamento

*e-mail: cleonia.araujo@univasf.edu.br é referente à tecnologia de fabricação do fármaco, segundo Vieira e Ohayon. ${ }^{1}$ Ainda segundo os mesmos autores, a margem bruta de lucro da industria farmacêutica estar entre $60 \%$ e $100 \%$, quando envolve tecnologia de descoberta de NEQ e drogas. Assim, entendendo a relevância da descoberta de NEQ, uma sequência didática exemplificando etapas deste processo foi planejada para ser empregada em cursos de graduação.

\section{ASPECTOS TEÓRICOS}

\section{A regra dos cinco de Lipinski}

Durante o desenvolvimento de fármacos o "Lead compound" (molécula com atividade farmacológica promissora que necessita de melhoramento) pode ter origem de uma fonte natural (vegetal ou animal), ou ser um produto de síntese química, item pertencente a uma coleção química, ou ainda, ser um protótipo molecular resultado de um planejamento racional para obtenção de fármacos. ${ }^{2-4}$ Independentemente de sua origem, a maioria dos "Lead compound" precisam ser melhorados através de modificações químicas até adquirirem propriedades farmacológicas aceitáveis, tais como: maior potência e seletividade, e menor toxicidade. ${ }^{5}$

No decorrer do processo de melhoramento de uma droga através de modificações químicas, muitas vezes, algumas características físico-químicas são drasticamente alteradas, gerando um comportamento farmacocinético indesejado, fato inclusive que pode tornar a droga inapropriada para administração por via oral. ${ }^{6}$ Em 1997 , Christopher A. Lipinski e colaboradores, trabalhando para a Indústria Farmacêutica Pfizer, realizaram um estudo com mais de 2000 fármacos, no qual observaram que algumas propriedades físico-químicas eram necessárias para que as drogas apresentassem boa solubilidade em água e permeabilidade intestinal, características essenciais para a disponibilidade oral. ${ }^{7}$ 
Os resultados alcançados pelo estudo liderado por Lipinski gerou a chamada regra dos cinco de Lipinski, regra dos cinco, ou "rule of five" (RO5). A RO5 tem o intuito de auxiliar os químicos medicinais nas decisões relacionadas às modificações químicas e, assim, reduzir o número de compostos preparados com propriedades físico-químicas indesejadas. De acordo com a RO5 são propriedades moleculares relevantes: o $\log$ P, o número de doadores de ligações de hidrogênio, o número de aceptores de ligações de hidrogênio, o peso molecular, bem como, os pontos de interação para os principais alvos de fármacos, como os ligantes de receptores acoplados à proteína $\mathrm{G}$, inibidores de cinases, moduladores de canais iônicos e receptores nucleares. ${ }^{8}$

A RO5 propõe que a má absorção ou permeação é mais provável quando a molécula possui mais que 5 doadores de ligação de hidrogênio, mais que 10 aceptores de ligação de hidrogênio, peso molecular maior do que 500 daltons e $\log \mathrm{P}$ calculado (Clog P) maior do que 5, sendo todos os parâmetros múltiplos de cinco. ${ }^{8}$

Extensões da RO5 foram criadas para melhorar as estimativas de toxicidade, desta forma, as moléculas promissoras a fármacos devem possuir $\log$ P na faixa de $-0,4$ a $+5,6$; refratividade molar de 40 a 130 $\mathrm{m}^{3} / \mathrm{mol}$; peso molecular de 180 a 500 daltons; número de átomos de 20 a 70; superfície polar não superior a $140 \hat{\mathrm{A}}^{2}$ e menos que 10 ligações rotativas. ${ }^{9}$ Considerando a relevância da disponibilidade das drogas durante os ensaios clínicos, bem como as vantagens da via oral para a administração de fármacos, é muito importante que durante o desenvolvimento de novas moléculas bioativas, seja verificado se a RO5 está sendo seguida ou violada em algum parâmetro.

\section{Heterociclos 1,3,4-oxadiazol}

Os heterociclos são compostos de importância indiscutível para a Química Medicinal, e trata-se de estruturas cíclicas constituída de carbono e pelo menos outro elemento diferente, sendo comumente o oxigênio, o nitrogênio e o enxofre, os heteroátomos. ${ }^{10,11}$ Os heterociclos estão presente em moléculas com diversas aplicações, dando destaque para produtos farmacêuticos, agroquímicos e veterinários. Também são utilizados como reveladores, antioxidantes, inibidores de corrosão, copolímeros, cosméticos, corantes, dentre outras aplicações. ${ }^{12}$

O grande interesse da indústria farmacêutica nestes compostos é resultado das diversas atividades biológicas que lhes são atribuídas, de modo que aproximadamente $62 \%$ dos fármacos empregados na terapêutica possuem heterociclos, bem como da diversidade estrutural que podem apresentar e de sua origem, a qual pode ser uma fonte natural ou sintética. ${ }^{13-16}$

Os oxadiazóis constituem uma classe importante de compostos heterocíclicos que contém um átomo de oxigênio e dois átomos de nitrogênio em um anel de cinco membros, com fórmula geral $\mathrm{C}_{2} \mathrm{H}_{2} \mathrm{ON}_{2} \cdot{ }^{17}$ São possíveis quatro isômeros do núcleo oxadiazol, que variam quanto à posição dos nitrogênios presentes no ciclo em relação ao oxigênio, sendo estes: i) 1,2,3-oxadiazol; ii) 1,2,4-oxadiazol; iii) 1,2,5 oxadiazol; e iv) 1,3,4-oxadiazol. ${ }^{18}$

Os 1,3,4-oxadiazóis quando substituídos nas posições 2 e 5 possuem ângulo aproximado de $134^{\circ}$ entre os substituintes e estabilidade térmica, o que torna este heterociclo promissor para a confecção de materiais funcionais avançados, que podem ser empregados na obtenção de dispositivos orgânicos, tais como: polímeros luminescentes, semicondutores, dispositivos emissores de luz e outros. ${ }^{19,20}$ Do ponto de vista da Química Medicinal, substâncias contendo este heterociclo podem possuir atividades biológicas de interesse, tais como: antitumoral, agonista de receptores benzodiazepínicos, analgésico, diurético, bactericida, dentre outros. ${ }^{21}$

A preparação do anel 1,3,4-oxadiazol pode acontecer via ciclodesidratação de diacil hidrazidas, ${ }^{22}$ oxidação de acil hidrazonas, ${ }^{23}$ reação entre acil hidrazida e ortoésteres, ${ }^{24}$ rearranjo de diaril-tetrazinas ${ }^{25}$ e reações entre tetrazóis e cloreto de acila (reação de Huisgen), ${ }^{26}$ Figura 1.

A oxidação de acil hidrazonas é uma das metodologias mais empregadas para obtenção de anéis 1,3,4-oxadiazóis na qual, em geral, são utilizadas quantidades estequiométricas de agentes oxidantes tais como $\mathrm{KMnO}_{4},\left(\mathrm{NH}_{4}\right)_{2}\left[\mathrm{Ce}\left(\mathrm{NO}_{3}\right)_{6}\right], \mathrm{Br}_{2}$, cloramina T, $\mathrm{FeCl}_{3}, \mathrm{HgO} / \mathrm{I}_{2}$, reagentes de chumbo tetravalentes ou reagentes de iodo hipervalentes. ${ }^{27}$

Sendo os heterociclos unidades estruturais comuns nos fármacos disponíveis, aproximadamente $80 \%$ dos medicamentos possuem este fragmento. Considerando a sua relevância para o desenvolvimento de novas moléculas bioativas, visto que os heterociclos são empregados como bioisosteros de diversos grupos funcionais, alterando propriedades das drogas, tais como seletividade, potência, lipofilicidade e solubilidade aquosa, ${ }^{28}$ destacamos a importância e corroboramos

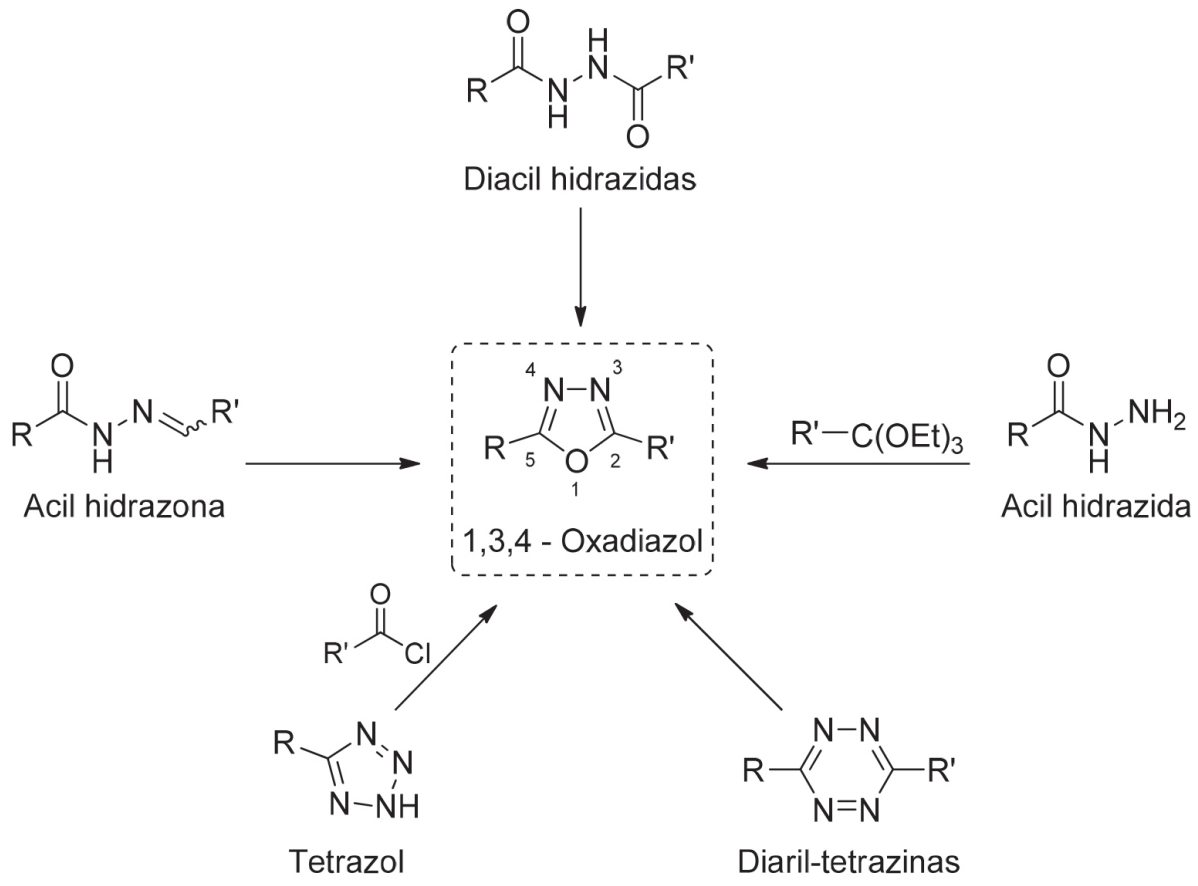

Figura 1. Vias sintéticas possíveis para obtenção do anel 1,3,4-oxadiazol 
com o desenvolvimento e divulgação de aulas contextualizadas de preparação de heterociclos em cursos de graduação, em disciplinas como Química Medicinal e Síntese Orgânica. ${ }^{29}$

\section{Irradiação de micro-ondas na síntese orgânica}

A química verde é um ramo da química que utiliza matéria-prima eficiente e preferencialmente de fonte renovável, eliminando a produção de resíduos e evitando o uso de reagentes e solventes tóxicos na produção e aplicação de produtos químicos..$^{30,31}$ A química verde é fundamentada em 12 princípios básicos que buscam primordialmente reduzir o desperdício, substituir o uso de substâncias perigosas e reduzir a energia utilizada pelos processos químicos, de modo que, quando possível, sejam conduzidos à temperatura e pressão ambientes..$^{31,32}$

O emprego da radiação de micro-ondas como fonte de aquecimento para reações químicas é um exemplo de aplicação da química verde no âmbito da síntese orgânica, uma vez que seu uso é capaz de diminuir drasticamente o tempo das reações, melhorar a seletividade, além de permitir a síntese de novas substâncias utilizando quantidades mínimas, ou até mesmo nenhum solvente. . $6,33,34^{-1}$

A radiação por micro-ondas é uma forma de energia eletromagnética, localizada entre a frequência do infravermelho e do rádio, entre 0,3 e $300 \mathrm{GHz}$, correspondente a ondas de comprimento de 1 $\mathrm{mm}$ a $1 \mathrm{~m} \cdot{ }^{30,35,36}$

A energia fornecida pelas micro-ondas é de aproximadamente $0,0016 \mathrm{eV}$, enquanto que as ligações químicas apresentam valores entre 0,04 e 7,71 eV. ${ }^{37}$ Deste modo, observa-se que as micro-ondas não são capazes de quebrar ligações químicas levando à excitação puramente cinética das moléculas, fato que favorece a ocorrência de reações sem alterar a estrutura molecular dos compostos reagentes e, consequentemente, sem promover a geração de subprodutos e/ou produtos de degradação. ${ }^{16,38}$

$\mathrm{O}$ aquecimento realizado pela radiação de micro-ondas fundamenta-se na geração de três efeitos principais, sendo estes: térmicos, específicos e não térmicos. Os efeitos térmicos são gerados em decorrência das altas temperaturas reacionais que podem ser alcançadas com o emprego de micro-ondas, e não são exclusivos deste tipo de radiação. Os efeitos específicos são aqueles produzidos unicamente pela radiação de micro-ondas, como por exemplo, o superaquecimento de solventes..$^{35}$ Já os efeitos não térmicos baseiam-se principalmente em dois fundamentos: o aumento da probabilidade de choques intermoleculares, devido às sucessivas mudanças de orientação das moléculas polares em função da oscilação do campo elétrico e a formação de espécies carregadas no estado de transição que são favorecidas pela radiação de micro-ondas, devido à interação com o campo elétrico gerado. ${ }^{37}$

Assim, os efeitos promovidos pela radiação de micro-ondas mostram-se vantajosos quando aplicados na síntese de compostos orgânicos, uma vez que são capazes de promover uma diminuição significativa dos tempos de reação e de favorecer a síntese de produtos de forma mais seletiva e, consequentemente, com rendimentos maiores quando comparado ao emprego do aquecimento convencional. .5,37,39 $^{35}$ Diante da importância de temas como desenvolvimento de NEQ, regra dos cindo de Linpiski, preparação de heterociclos e o emprego de energia de micro-ondas em síntese orgânica, o presente trabalho trata de uma sequencia didática que contextualiza o planejamento de moléculas bioativas, mostrando inicialmente a importância da determinação teórica de propriedades das moléculas, introduzindo ao estudante o conceito de regra dos cinco de Lipinski e dando sequencia com a preparação de uma substância contendo o ciclo 1,3,4-oxadiazol utilizando um aparelho de micro-ondas doméstico.

\section{PARTE EXPERIMENTAL}

\section{Planejamento da sequência didática}

A sequência didática aqui apresentada foi planejada para ser aplicada em cursos de graduação onde haja o interesse pelo desenvolvimento de NEQ, como os cursos de Farmácia, Química e afins. Os conteúdos serão expostos de maneira a fornecer ao discente, de forma simplificada e conciliando um pouco da teoria com a prática, as etapas do desenvolvimento de novos fármacos. Desta forma, dentro do conteúdo de desenvolvimento de fármacos, o docente deverá apresentar a relevância da presença de heterociclos em moléculas bioativas e a importância do planejamento farmacocinético, dando ênfase à regra dos cinco de Lipinski.

Na primeira etapa da aula os alunos utilizarão uma base de dados de livre acesso, o ChemSpider $^{\circledR}$, na qual poderão prever propriedades químicas relevantes para moléculas promissoras do ponto de vista farmacológico. No segundo momento, os estudantes prepararão um composto heterocíclico de 5 membros, a partir da isoniazida (1) e do benzaldeído (2), em uma rota sintética de duas etapas e empregando um aparelho de micro-ondas doméstico (Figura 2).

Os autores sugerem a divisão do experimento em três aulas práticas de $2 \mathrm{~h}$ cada, sendo a primeira destinada ao uso do software ChemSpider $^{\circledR}$ pelos estudantes, a partir do qual os mesmos irão avaliar se o protótipo heterocíclico 1,3,4-oxadiazol (4) se adequa ou não à RO5. A segunda aula destinada à realização da reação de condensação entre a isoniazida (1) e o benzaldéido (2) para obtenção do intermediário sintético, a acil-hidrazona (3), seguido de sua caracterização por determinação de ponto de fusão (PF) e cromatografia líquida em camada delgada (CCD). Por fim, a terceira aula prática, destinada à síntese do composto heterocíclico 1,3,4-oxadiazol (4), por meio da ciclização oxidativa da acil-hidrazona (3) preparada na aula anterior, seguido de sua caracterização por PF, CCD e espectroscopia de ressonância magnética nuclear (RMN).

\section{Aula Prática 1: Aplicação da regra dos cinco de Lipinski utilizando o ChemSpider $^{\circledR}$}

Sugere-se que a primeira atividade ocorra no laboratório de informática da instituição. Nesta aula, os estudantes utilizarão o software gratuito ChemSpider ${ }^{\circledR}$ para verificar se a estrutura molecular do 2-fenil-5-(4piridil)-1,3,4-oxadiazol (4) que os estudantes irão sintetizar em uma aula prática posterior, demonstra alguma violação à RO5.

\section{Aula Prática 2: Síntese do intermediário sintético acil- hidrazona (3)}

\section{Reagentes e equipamentos}

Para as aulas práticas de síntese, todos reagentes foram obtidos comercialmente e utilizados sem purificação prévia. O aparelho de

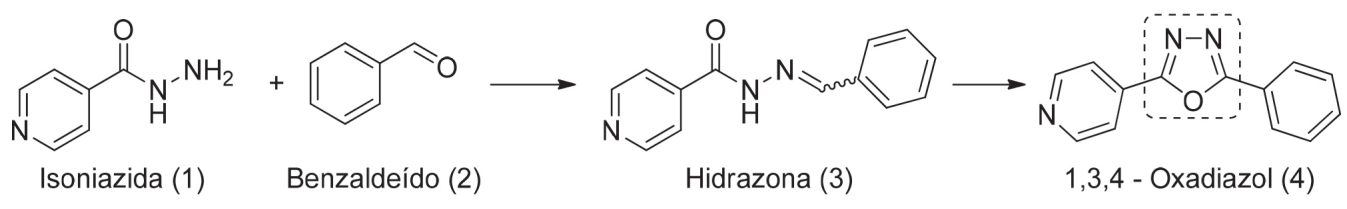

Figura 2. Esquema reacional para a obtenção do 2-fenil-5-(4piridil)-1,3,4-oxadiazol (4) 
micro-ondas utilizado foi de uso doméstico (Electrolux ${ }^{\circledR}$, modelo MEG41 com potência ajustável até 1500 W). As substâncias obtidas tiveram seus pontos de fusão determinados empregando-se um fusiômetro (Tecnopon, modelo PFM II). Os espectros de RMN de ${ }^{1} \mathrm{H}$ foram registrados sob $400 \mathrm{MHz}$ em solvente deuterado $\left(\mathrm{CDCl}_{3}\right)$ utilizando um espectrômetro (Bruker ${ }^{\circledR}$, modelo Ascend-400 MHz) e os deslocamentos químicos $(\delta)$ estão em partes por milhão (ppm) em relação ao padrão interno tetrametilsilano (TMS). Já os espectros de $\mathrm{RMN}$ de ${ }^{13} \mathrm{C}$ e Dept-135 foram registrados de forma similar ao anterior, estando o espectrômetro operando na frequência de100 MHz.

\section{Síntese do intermediário}

Para a síntese do intermediário acil-hidrazona (3) foram misturados em um balão de reação de $50 \mathrm{~mL}: 110 \mu \mathrm{L}$ de benzaldeído (1) (1 $\mathrm{mmol}, \mathrm{d}=1,04 \mathrm{~g} / \mathrm{mL}), 137 \mathrm{mg}$ de isoniazida (2) (1 mmol), $4 \mathrm{~mL}$ de etanol absoluto e 3 gotas de $\mathrm{H}_{3} \mathrm{PO}_{4}$ concentrado. A mistura reacional foi submetida à irradiação de micro-ondas, utilizando um aparelho doméstico, com potência de $300 \mathrm{~W}$ (potência ajustada de 20\%) por 15 min, pausado em intervalos de 30 segundos. Um funil de vidro invertido $(\mathrm{di}=80 \mathrm{~mm}$ ) foi colocado sobre a boca do balão de reação para minimizar a perda do solvente. Ao fim deste período, observou-se a formação de pequena quantidade de um sólido amarelo que foi separado do solvente por filtração a vácuo e descartado. O solvente foi removido em evaporador rotativo, obtendo-se $218 \mathrm{mg}$ de um sólido de coloração bege com PF:207-212 ${ }^{\circ} \mathrm{C}$ (PF Lit.:196-198 ${ }^{\circ} \mathrm{C}$ ).

\section{Aula Prática 3: Síntese do composto heterocíclico 1,3,4-oxadiazol (4)}

\section{Síntese do produto final}

Para a síntese do heterociclo 1,3,4-oxadiazol, o 2-fenil-5-(4-piridil)-1,3,4-oxadiazol (4), foram misturados em um balão de reação de $50 \mathrm{~mL}$ : $180 \mathrm{mg}$ da acil-hidrazona $(3)(0,8 \mathrm{mmol})$ preparada na aula prática anterior, $243 \mathrm{mg}$ de $\mathrm{I}_{2}(0,96 \mathrm{mmol}), 331 \mathrm{mg}$ de $\mathrm{K}_{2} \mathrm{CO}_{3}$ $(2,4 \mathrm{mmol})$ e $3 \mathrm{~mL}$ de DMSO. A mistura reacional foi submetida à irradiação de micro-ondas, utilizando um aparelho doméstico, com potência de $750 \mathrm{~W}$ (potência ajustada de $50 \%$ ) por $10 \mathrm{~min}$, pausado em intervalos de 20 segundos. Ao fim deste período, a reação foi deixada esfriar até temperatura ambiente e, em seguida, foi adicionado $20 \mathrm{~mL}$ de uma solução aquosa a $5,0 \%(\mathrm{~m} / \mathrm{v})$ de $\mathrm{Na}_{2} \mathrm{~S}_{2} \mathrm{O}_{3} \cdot 5 \mathrm{H}_{2} \mathrm{O}$. O produto foi extraído do meio reacional através de uma extração líquido-líquido, empregando acetato de etila ( 3 x $10 \mathrm{~mL})$. A fase orgânica foi lavada com água deionizada $(3 \times 10 \mathrm{~mL}) \mathrm{e}$, em seguida, tratada com $\mathrm{Na}_{2} \mathrm{SO}_{4}$ anidro e o solvente removido em evaporador rotativo, obtendo-se 189 $\mathrm{mg}(0,84 \mathrm{mmol})$, rendimento de $84 \%$, de um sólido de coloração bege. Este foi caracterizado sem purificação adicional. $\mathrm{PF}: 154-158^{\circ} \mathrm{C}(\mathrm{PF}$ Lit.: $\left.152-155{ }^{\circ} \mathrm{C}\right), \mathrm{RMN}$ de ${ }^{1} \mathrm{H}\left(\mathrm{CDCl}_{3}\right)$ : 7,67 (m; $\left.3 \mathrm{H} \mathrm{Ar}\right)$; 8,02 (m; $2 \mathrm{H} \mathrm{Ar}) ; 8,17$ (m; 2H Ar); 8,86 (m; $2 \mathrm{H} \mathrm{Ar})$; RMN de ${ }^{13} \mathrm{C}\left(\mathrm{CDCl}_{3}\right)$ : 120,$36 ; 123,30 ; 127,15 ; 129,22 ; 131,11 ; 132,31 ; 150,77 ; 162,71$ (C anel heterociclo); 165,49 (C anel heterociclo); Dept-135 $\left(\mathrm{CDCl}_{3}\right)$ : 120,$43 ; 127,22 ; 129,42 ; 132,48 ; 150,96$.

\section{Caracterização por cromatografia em camada delgada}

Amostras de isoniazida (1), benzaldeído (2), produto da reação de condensação (3) e produto da reação de ciclização (4) foram dissolvidas em etanol absoluto e aplicadas lado a lado numa placa de alumínio $(2 \mathrm{~cm}$ x $4 \mathrm{~cm}$ ) recoberta com sílica em gel 60 contendo indicador fluorescente $\mathrm{GF}_{254}$. Em seguida, a placa foi eluida empregando-se o sistema de solvente acetato de etila/metanol (AcEt/ $\mathrm{MeOH}$ ) na proporção 9:1. A placa cromatográfica foi revelada com luz ultravioleta, nos comprimentos de onda de 254 e $365 \mathrm{~nm}$ e os fatores de retenção (Rf) das manchas reveladas foram medidos para fins de comparação.

\section{RESULTADOS E DISCUSSÃO}

A proposta apresentada visa contextualizar aulas de disciplinas de cursos de graduação que tratem do desenvolvimento de NEQ, tais como: Química Farmacêutica; Química Medicinal; Síntese de Fármacos e outras, buscando trazer para a realidade das salas de aula tópicos que são relevantes para a $\mathrm{P} \& \mathrm{D}$ da indústria farmacêutica. A sequência didática é composta por atividades práticas computacionais e experimentais, em que inicialmente os estudantes serão apresentados à RO5 e, utilizando o ChemSpider ${ }^{\circledR}$, irão conhecer uma maneira de verificar se determinada molécula viola ou não essa regra. As etapas experimentais de laboratório tratam-se da síntese de um composto heterocíclico 1,3,4-oxadiazol, uma estrutura relativamente complexa, empregando métodos sintéticos simples, de baixo custo e altos rendimentos, características almejadas pela indústria farmacêutica.

O ChemSpider ${ }^{\circledR}$ é uma base de dados de acesso livre que pertence à Royal Society of Chemistry, e fornece variadas informações de mais de 58 milhões de substâncias químicas a partir de aproximadamente 500 fontes de dados. ${ }^{40}$ Esta ferramenta permite a busca por um termo, que pode ser o nome de compostos de interesse, sinônimos, número de registro da substância (CAS) ou outros. O que torna a plataforma ainda mais interessante é que a pesquisa também pode ser realizada através da busca pela estrutura química da substância que está em estudo.

Dentre as informações fornecidas pela base de dados, como resultado de busca, estão as estimativas das propriedades físico-químicas realizadas com o ACD/Labs, EPISuite, ChemAxon e Mcule. Nesta atividade didática foram utilizados os dados gerados pelo $A C D / L a b s$, pois é realizada gratuitamente e sem necessidade de cadastro prévio. Diante do exposto, a primeira aula prática foi ministrada no laboratório de informática da instituição e os estudantes foram orientados a acessar o ChemSpider ${ }^{\circledR}$ através do endereço: http://www.chemspider. $\mathrm{com} /$. E em seguida, clicar no link Structure, para abrir uma página que permite desenhar estruturas químicas moleculares. A estrutura do 2-fenil-5-(4-piridil)-1,3,4-oxadiazol (4) foi desenhada e a busca realizada através do link Search. Neste momento foi visualizada a estrutura com seu nome e sinônimos, os alunos foram orientados a abrir a aba Properties e, em seguida, Predicted Advanced Chemistry Development (ACD/Labs). Um quadro com as propriedades do composto heterocíclico foi gerado e as de interesse que estavam relacionadas com a RO5 de Lipinski foram avaliadas. Foi observado dentre os parâmetros analisados que a regra dos cinco não foi violada em \#Rule of 5 Violations. Em seguida, o professor foi indicando cada propriedade obtida com os parâmetros da RO5, explicando que a não violação destes é relevante para a farmacocinética de moléculas com atividade biológica promissora, Tabela 1 .

Tabela 1. Propriedades do 2-fenil-5-(4-piridil)-1,3,4-oxadiazol (4) obtidas através do $A C D / L a b s$

\begin{tabular}{lc}
\hline PROPRIEDADE & VALOR \\
\hline Massa molar & $223.230 \mathrm{Da}$ \\
Número de átomos & $26^{\mathrm{a}}$ \\
Refratividade molar & $62.0 \pm 0.3 \mathrm{~cm}^{3}$ \\
Aceptores de H em ligações de hidrogênio & 4 \\
Doadores de H em ligações de hidrogênio & 0 \\
Ligações com rotação livre & 2 \\
LogP & 2.89 \\
Área superficial polar & $52 \hat{\mathrm{A}}^{2}$ \\
\hline
\end{tabular}

aSoma do número de átomos disponível na fórmula molecular. 
Dando sequência à aula, o professor estimulou os estudantes a propor estruturas análogas do 2-fenil-5-(4-piridil)-1,3,4-oxadiazol (4) e verificar, via ChemSpider $^{\circledR}$, se havia ocorrido violação da RO5 e qual teria sido a(s) propriedade(s) que estava $(\mathrm{m})$ fora da regra. Caso o docente tenha preferência pelo Scifinder ${ }^{\circledR}$, base de dados oferecida pela American Chemical Society, a ferramenta também estima as propriedades empregando o $\mathrm{ACD} / \mathrm{Labs}$, contudo é necessário realizar um cadastro prévio. ${ }^{41}$

O segundo momento da sequência didática ocorreu no laboratório de química. Neste encontro foi realizada a reação de condensação entre uma hidrazida e um aldeído para obtenção da acil-hidrazona (3) ${ }^{39}$ Para esta reação, a isoniazida (1) foi colocada para reagir com o aldeído aromático benzaldeído (2), em meio de etanol absoluto e empregando-se $\mathrm{H}_{3} \mathrm{PO}_{4}$ como catalisador e sob irradiação de micro-ondas. A mistura reacional foi submetida à irradiação por micro-ondas na potência de $300 \mathrm{~W}$, sendo empregado um aparelho de micro-ondas de uso doméstico, ajustado na potência 20, a qual corresponde a $20 \%$ da potência máxima $(1500 \mathrm{~W})$ do eletrodoméstico. ${ }^{42} \mathrm{~A}$ acil-hidrazona (3) foi obtida como um sólido de coloração bege, com ótimo rendimento reacional (97\%), apresentando faixa de fusão de $207-212{ }^{\circ} \mathrm{C}$, a qual corroborou com a faixa de fusão descrita na literatura (PF: 196-198 ${ }^{\circ} \mathrm{C}$ ) para esta molécula. ${ }^{43} \mathrm{Na}$ caracterização por CCD (Figura 1S), foi observado que o produto obtido apresentou $\mathrm{Rf}$ diferente dos precursores de sua síntese. A isoniazida (1), o benzaldeído (2) e a acil-hidrazona (3), revelaram os seguintes valores de Rf: 0,17; 0,94 e 0,47 no sistema de solvente AcEt/MeOH (9:1). Este resultado, além de reforçar a confirmação da preparação da acil-hidrazona (3), também demonstrou que o produto de síntese estava puro.

A escolha da isoniazida (2), um fármaco comercial, componente da terapia farmacológica da tuberculose, como material de partida foi a maneira encontrada pelos autores para mostrar que a escassez de recursos não pode limitar o desenvolvimento de aulas contextualizadas e que abordem conhecimentos atuais. ${ }^{44-47}$ Além disso, o emprego da isoniazida (2) destaca uma tendência da indústria farmacêutica em empregar moléculas bioativas já estudadas no desenvolvimento de NEQ, uma vez que, iniciar estudos científicos partindo de moléculas que possuem propriedades químicas, físico-químicas, farmacológicas, toxicológicas e farmacocinéticas conhecidas pode ser vantajoso. ${ }^{2}$

O uso de aparelho de micro-ondas doméstico possibilitou a obtenção da hidrazona (3), com ótimo rendimento e com um curto tempo de reação, tendo em vista que a síntese se processa por completo em um período de 15 minutos. $\mathrm{O}$ aumento da velocidade e a consequente diminuição do tempo de reação deve-se a combinação dos efeitos (térmicos, específicos e não térmicos) resultantes da irradiação por micro-ondas, os quais são capazes de promover um aumento no grau de agitação das moléculas, fazendo com que energia térmica seja gerada por fricção. ${ }^{48}$ Além disso, em reações realizadas em fornos de micro-ondas a energia é transferida diretamente aos reagentes, enquanto que no aquecimento por métodos convencionais, utilizando chapas aquecedoras, por exemplo, a transferência de energia ocorre por convecção, de forma que esta é transferida lentamente do recipiente para o meio reacional..$^{35}$

$\mathrm{Na}$ terceira aula experimental foi dada continuidade à rota sintética para obtenção do heterociclo 1,3,4-oxadiazol (4), sendo o mesmo obtido através da ciclização oxidativa da acil-hidrazona (3), preparada na aula prática anterior, empregando-se iodo molecular como agente oxidante. ${ }^{27}$ Para esta síntese a fonte de calor convencional foi alterada, sendo também utilizada a irradiação de micro-ondas e, como na etapa anterior, o heterociclo foi obtido com um tempo de reação consideravelmente curto (10 minutos) e com alto rendimento (84\%). A determinação do ponto de fusão permitiu evidenciar que o protótipo 1,3,4-oxadiazol (4) foi sintetizado, uma vez que apresentou faixa de fusão $\left(154-157^{\circ} \mathrm{C}\right)$ semelhante à descrita na literatura para esta molécula $\left(152-155^{\circ} \mathrm{C}\right) .{ }^{49} \mathrm{O}$ sucesso da etapa reacional foi inicialmente analisado por CCD (Figura 1S), a partir da qual foi observado que o produto gerado possui um $\mathrm{Rf}$ de 0,73 , valor diferente dos $\mathrm{Rfs}$ dos reagentes e do intermediário sintético, considerando o sistema de solvente usado para eluição AcEt/MeOH (9:1). Além disso, ainda na CCD, também foi observado que o produto da ciclização emite fluorescência azul ao ser irradiado com luz UV de $365 \mathrm{~nm}$. Esta é uma característica resultante da deficiência eletrônica, do elevado rendimento quântico de fotoluminescência e da estabilidade química do grupo 1,3,4-oxadiazol, já relatada na literatura. ${ }^{50,51}$ Por fim, para elucidar a estrutura do produto sintetizado foram realizadas análises dos espectros de RMN de ${ }^{1} \mathrm{H},{ }^{13} \mathrm{C}$ e Dept-135 (Figuras 2S, 3S e 4S), as quais confirmaram o sucesso da metodologia empregada para a síntese do composto heterocíclico 1,3,4-oxadiazol (4) utilizando aparelho de micro-ondas doméstico.

O método empregado para realizar a ciclização oxidativa, ao contrário dos métodos convencionais, não utiliza metais, o que o torna vantajoso para a indústria farmacêutica, pois não gera impurezas metálicas, tem baixo custo e possui processos operacionais simples. Novamente foi possível observar a influência da irradiação de micro-ondas, a qual viabilizou a obtenção do composto heterocíclico em tempo hábil e com rendimento satisfatório para a execução de uma aula prática de graduação. As aulas práticas de síntese orgânica usando micro-ondas também demonstraram a aplicação de metodologias que condizem com os princípios da química verde, já que o tempo reacional e a quantidade de solvente (DMSO) utilizado foram reduzidos, comparando-se tais parâmetros com os normalmente observados nas metodologias tradicionais. ${ }^{27}$

\section{CONCLUSÃO}

A sequência didática apresentada possibilitou aos estudantes uma breve contextualização de algumas etapas do desenvolvimento de fármacos utilizadas no setor de $\mathrm{P} \& \mathrm{D}$ da indústria farmacêutica, demonstrando uma aula prática relacionada com o planejamento de novos fármacos, através da aplicação da regra dos cinco de Lipinski com auxílio do software ChemSpider ${ }^{\oplus}$, e outras aulas abordando a produção de uma NEQ, com a síntese do composto heterocíclico 1,3,4-oxadiazol. A verificação da concordância da estrutura química do oxadiazol proposto com a RO5 demonstrou sua viabilidade como uma molécula com possível atividade biológica, assim como, permitiu aos estudantes compreender de forma didática a importância dos descritores para a farmacocinética de NEQ. A síntese do composto heterocíclico 1,3,4-oxadiazol mostrou-se aplicável ao ensino de síntese orgânica em cursos de graduação, uma vez que este produto pôde ser obtido em curto tempo de reação, alto rendimento e com a mínima produção de resíduo. A utilização de um aparelho de forno micro-ondas doméstico como fonte de aquecimento também contribuiu para a viabilidade desta síntese em laboratórios de universidades e faculdades que não possuem o aparelho específico de alto custo. Além disso, esta sequência didática também pode ser utilizada como ferramenta introdutória do conceito e dos princípios da química verde, bem como do uso de técnicas modernas na síntese de compostos heterocíclicos para estudantes de graduação.

\section{MATERIAL SUPLEMENTAR}

As imagens de CCD realizadas bem como os espectros de RMN de ${ }^{1} \mathrm{H},{ }^{13} \mathrm{C}$ e Dept-135 do composto heterocíclico sintetizado neste trabalho estão disponíveis em http://quimicanova.sbq.org.br, na forma de arquivo PDF, com acesso livre. 


\section{AGRADECIMENTOS}

\section{Ao CNPq, à FACEPE e à UNIVASF.}

\section{REFERÊNCIAS}

1. Vieira, V. M. M.; Ohayon, P.; Revista Economia \& Gestão 2006, 13, 1.

2. Aver, G. M.; Kreutz, O. C.; Suyenaga, E. S.; Revista Conhecimento Online 2015, 2, 63.

3. Guido, R. V. C.; Andricopulo, A. D.; Oliva, G.; Estudos Avançados 2010, 24,81 .

4. Sangi, D. P.; Quim. Nova 2016, 39, 995.

5. Lima, L. M.; Quim. Nova 2007, 30, 1456.

6. Manly, C. J.; Chandrasekhar, J.; Ochterski1, J. W.; Hammer, J. D.; Warfield, B. B.; Drug Discovery Today 2008, 13, 99.

7. Lipinski, C. A.; Drug Discovery Today: Technol. 2004, 4, 337.

8. Lipinski, C. A.; Lombardo, F.; Dominy, B. W.; Feeney, P. J.; Adv. Drug Delivery Rev. 2001, 46, 3.

9. Veber, D. F.; Johnson, S. R.; Cheng, H. Y.; Smith, B. R.; Ward, K. W.; Kopple, K. D. J.; Med. Chem. 2002, 45, 2615.

10. International Union of Pure and Applied Chemistry (IUPAC); Compendium of Chemical Terminology, $2^{\text {nd }}$ ed, Blackwell Scientific Publications: Oxford, 1997.

11. Martins, P.; Jesus, J.; Santos, S.; Raposo, L. R.; Roma-Rodrigues, C.; Baptista, P. V.; Fernandes, A. R.; Molecules 2015, 20, 16852.

12. Arora, P.; Arora, V.; Lamba, H. S.; Wadhwa, D.; Int. J. Pharm. Sci. Res. 2012, 3, 2947.

13. Menegatti, R.; Fraga, C. A. M.; Barreiro, E. J.; Cad. Tematicos Quim. Nova Esc. 2001, 3, 16

14. Dua, R.; Shrivastava, S.; Sonwane, S. K.; Srivastava, S. K.; Adv. Biol. Res. 2011, 5, 120.

15. Kaushik, N. K.; Kaushik, N.; Attri, P.; Kumar, N.; Kim, C. H.; Verma A. K.; Choi, E. H.; Molecules 2013, 18, 6620.

16. Duarte, P. D.; Sangi, D. P.; Corrêa, A. G.; Rev. Virtual Quim. 2010, 2 , 204.

17. Kavitha, S.; Gnanavel, S.; Kannan, K.; Asian J. Pharm. Clin. Res. 2014, $7,11$.

18. Salahuddin; Mazumder, A.; Shaharyar, M.; BioMed Res. Int. 2014, 2014, 1.

19. Schulz, B.; Orgzall, I.; Freydank, A.; Xue, C.; Adv. Colloid Interface Sci. 2005, 116, 143.

20. Hernandez-Ainsa, S.; Barbera, J.; Marcos, M.; Serrano, J. L.; Macromolecules (Washington, DC, U. S.) 2012, 45, 1006.

21. Majji, G.; Rout, S. K.; Guin, S.; Gogoi, A.; Patel, B. K.; RSC Adv. 2014, 4, 5357.

22. Lei, Z.-Y.; Kuo, H.-M.; Lai, C. K.; Tetrahedron 2017, 73, 1650.

23. Rao, V. S.; Sekhar, V. G. C.; Synth. Commun. 2004, 34, 2153.
24. Dabiri, M.; Salehi, P.; Baghbanzedeh, M.; Zolfigol, M. A.; Bahramnejad, M.; Synth. Commun. 2007, 37, 1201.

25. Goswami S.; Jena, H. S.; Konar, S. Inorg. Chem. 2014, 53, 7071.

26. Yang, L.; Guan, M.; Bian, Z.; Xie, J.; Chen, T.; Huang, C.; Thin Solid Films 2006, 500, 224.

27. Yu, W.; Huang, G.; Zhang, Y.; Liu, H.; Dong, L.; Yu, X.; Li, Y.; Chang, J.; J. Org. Chem. 2013, 78, 10337.

28. Gomtsyan, A. Chem. Heterocycl. Compd. 2012, $48,7$.

29. Cunha, S.; Santos-Filho, R. F.; Riatto, V. B. Quim. Nova 2013, 36, 190.

30. Jain, A. K.; Singla, R. K.; webmedcentral.com :: Pharmaceutical Sciences 2011, 2, 1.

31. Anastas, P. T.; Warner, J.; Green Chemistry: Theory and Practice; Oxford University Press: Oxford, 1998.

32. Tsukui, A.; Rezende, C. M.; Rev. Virtual Quim. 2014, 6, 1713.

33. Majumder, A.; Ragini, G.; Jain, A.; Green Chem. Lett. Rev. 2013, 6, 151.

34. Ravichandran, S.; Karthikeyan, E.; Int. J. ChemTech Res. 2011, 3, 466.

35. Teixeira, E. F.; Santos, A. P. B.; Bastos, R. S.; Pinto, A. C.; Kümmerle, A. E.; Coelho, R. R.; Quim. Nova 2010, 33, 1603.

36. Belwal, S.; Mod. Chem. 2013, 1, 22.

37. Souza, R. O. M. A.; Miranda, L. S. M.; Quim. Nova 2011, 34, 497.

38. Gangrade, D.; Lad, S. D.; Mehta A. L.; Int. J. Res. Pharm. Sci. 2015, 2 , 37.

39. Youssef, M. M.; Amin, M. A.; Molecules 2010, 15, 8827.

40. ChemSpider - Disponível em: http://www.chemspider.com/, acessado em setembro de 2017

41. Araújo, C. R. M.; Leite-Filho, C. A.; Santos, V. L. A.; Maia, G. L. A.; Gonsalves, A. A. Quim. Nova 2015, 38, 868

42. Rostamizadeh, S.; Housaini, S. A. G.; Tetrahedron Lett. 2004, 45, 8753.

43. Thomas, A. B.; Tupe, P. N.; Badhe, R. V.; Nanda, R. K.; Kothapalli, L. P.; Paradkar, O. D.; Sharma, P. A.; Deshpande, A. D.; Green Chem. Lett. Rev. 2009, 2, 23.

44. Cunha, S.; Santana, L. L. B.; Quim. Nova 2012, 35, 642.

45. Cunha, S.; Lustosa, D. M.; Conceição, N. D.; Quim. Nova 2012, 35, 638.

46. Konrath, E. L.; Piedade, M.; Eifler-Lima, V. L.; Quim. Nova 2012, 35, 1887.

47. Regueira, J. L. L. F.; de Freitas, J. J. R.; Freitas-Filho, J. R.; Quim. Nova 2016, 39, 1019 .

48. Sauer, A. C.; Dissertação de Mestrado, Universidade Federal de Santa Maria, Brasil, 2013.

49. Navarrete-Vázquez, G.; Molina-Salinas, G. M.; Duarte-Fajardo, Z. V.; Vargas-Villarreal, J.; Estrada-Soto, S.; González-Salazar, F.; HernándezNúnez, E.; Said-Fernández, S.; Bioorg. Med. Chem. 2007, 15, 5502.

50. Cristiano, R.; Dissertação de Mestrado, Universidade Federal de Santa Catarina, Brasil, 2004.

51. Neves-Filho, R. A. W.; Dissertação de Mestrado, Universidade Federal de Pernambuco, Brasil, 2008. 